Sir,

\section{Response to Bunce et al}

We read with interest the study of Causes of blind and partial sight certifications in England and Wales: April 2007-March 2008. ${ }^{1}$ It was stated that 'The numbers of certificates are also lower than the figures reported as registered at Social Services'. This finding was interesting to us as we had noted a similar problem while performing a cross-sectional study of all Certificates of Vision Impairment (CVI) for Bristol and North Somerset between September 2005 and September 2008. We found that 54 patients were registered separately at both Bristol and North Somerset Social Services. All these 54 patients had been referred from North Somerset into Bristol for sub-specialty ophthalmology assessment and had a CVI completed in Bristol Eye Hospital. The patient was entered onto the Bristol Social Services register at that visit. The CVI was later forwarded to North Somerset Social Services and entered onto their register. The patient was not deleted from the Bristol register and therefore these patients, representing $5.3 \%$ of all patients in our study, would be counted twice when the individual Social Services figures were forwarded to The Health and Social Care Information Centre. However, due to the way the CVI forms are processed locally, only one copy of the CVI form would be forwarded to the Certifications Office in London. If this problem was duplicated around other regions in England and Wales, then it may explain a proportion of the discrepancy noted by Bunce et al.

\section{Conflict of interest}

The authors declare no conflict of interest.

\section{Reference}

1 Bunce C, Xing W, Wormald R. Causes of blind and partial sight certifications in England and Wales: April 2007-March 2008. Eye 2010; 24: 1692-1699.

MJ Wakefield, DM Tole, CC Bailey, MV Mundasad and JM Sparrow

Department of Ophthalmology, Bristol Eye

Hospital, Bristol, UK

E-mail: mjwakefield_uk@hotmail.com

Eye (2011) 25, 821; doi:10.1038/eye.2011.20; published online 25 February 2011

Sir,

\section{Response to Wakefield et al}

We thank Wakefield et $a l^{1}$ for their interest in our paper ${ }^{2}$ and for a possible explanation regarding the discrepancy between certification and registration figures. An additional reason is that, despite much publicity, some Eye units are not using the correct address for the Certifications Office. It is important to note that we would not expect an exact match between certification and registration figures for the same time period, as there can be some delay between certification and registration.
We believe, however, that increasing use of an electronic alternative will resolve these issues and lead to high-quality data being readily available on the numbers newly certified by cause.

\section{Conflict of interest}

The authors declare no conflict of interest.

\section{References}

1 Wakefield MJ, Tole DM, Bailey CC, Mundasad MV, Sparrow JM. Response to Bunce et al. Eye 2011; 25: 821.

2 Bunce C, Xing W, Wormald R. Causes of blind and partial sight certifications in England and Wales: April 2007-March 2008. Eye 2010; 24: 1692-1699.

\section{Bunce, W Xing and R Wormald}

Research \& Development, Moorfields Eye Hospital, London, UK

E-mail: c.bunce@ucl.ac.uk

Eye (2011) 25, 821; doi:10.1038/eye.2011.21; published online 25 February 2011

Sir,

Cystoid macular oedema successfully treated by cryotherapy in retinitis pigmentosa with Coats'-like retinal exudation

The association between RP and exudative retinopathy was first reported by G. Zamorani in $1956 .{ }^{1}$ Coats'-like retinal changes are seen in 1-3\% of patients affected by RP. ${ }^{2}$ The aetiology is unknown. Mutations in the Crumbs homologue 1 (CRB1) gene have been reported $^{3}$ as a risk factor for developing Coats'-like changes in patients with autosomal recessive RP.

We present a case of a young patient with Coats'-like autosomal dominant retinitis pigmentosa (ADRP), exudative retinal detachment (E-RD), and cystoid macular oedema (CME), successfully treated with cryotherapy.

\section{Case report}

A 10-year-old boy presented at the Retina Clinic in Southampton Eye Unit with marked deterioration in visual acuity (VA) in his right eye. VAs were 0.67 $\log$ MAR OD and $0.14 \log$ MAR OS. There was a family history of ADRP (Figure 1). RP was diagnosed in the proband by retinal findings (Figure 2) and by electrophysiology performed 7 years before presentation (ERG, VEP). Mixed cone/rod ERGs as well as independent testing of the cone and rod-mediated responses were of borderline amplitude and binocular occipital pattern VEPs were present to the smallest checks. No funding for genetic testing for RP was available for any of the patient's affected relatives. In the right eye fundoscopy demonstrated mild vitreous haze, 'bone spicule like' pigment accumulation in the periphery bilaterally, Coats'-like E-RD, CME, teleangectatic vessels, and preretinal haemorrhages in the infero-temporal quadrant. 
CME had been treated previously with Acetazolamide $250 \mathrm{mg}$ bid for 3 months. There was no significant improvement. Similarly, neither Dorzolamide hydrochloride drops tid for another 3 months or subsequently an orbital floor injection of Depo-Medrone $(40 \mathrm{mg})$ were effective.

On 3D-OCT retinal oedema and increased retinal thickness were present in the right macula (Figure 3a). OS OCT findings were unremarkable. Cryotherapy was applied to the infero-temporal quadrant. At 6 months after treatment, VA OD increased slightly to 0.62 $\log$ MAR, while 3D-OCT showed very mild residual CME (Figure 3b).

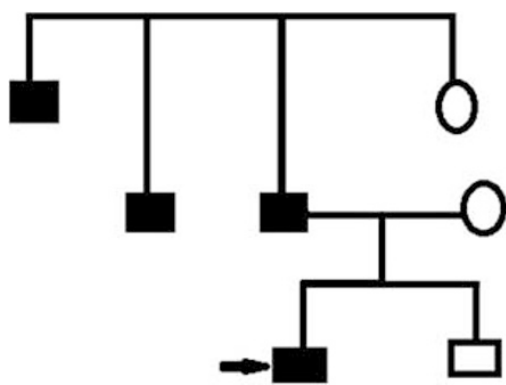

Figure 1 Patient's pedigree demonstrating autosomal dominant inheritance of RP in the family.

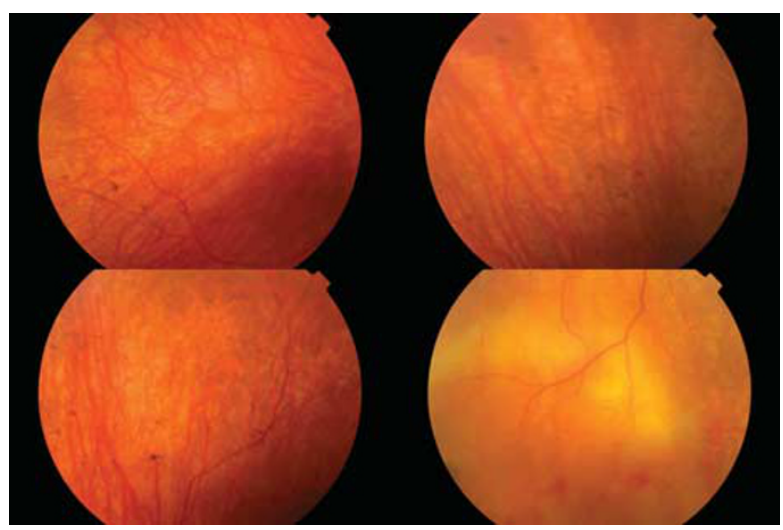

Figure 2 Colour fundus photograph of the right eye demonstrating exudative retinal detachment in the infero-temporal quadrant. Bone spicule like pigmentation, retinal exudates, teleangectatic vessels, and superficial haemorrhages are also present.

\section{Comments}

This is an unusual ADRP-Coats'-like case of unilateral retinal involvement. Coats reactions are infrequently seen in ADRP patients.

Lee et $^{4} \mathrm{l}^{4}$ tried vitrectomy, drainage of subretinal fluid, cryotherapy, endolaser, and silicone oil tamponade in a similar case in the past. The detachment was successfully treated, but VA did not improve. Laser photocoagulation as used by Kan et $a l^{5}$ resulted in a slight reduction of the exudation.

We treated our patient on the basis that CME developed because of inflammation generated from this area of Coats'like exudation. Our hypothesis appeared correct, as cryotherapy alone led to complete resolution of the detachment with a resulting increase in VA and minimal residual CME. We suggest cryotherapy to be considered as an effective treatment option in the management of E-RD and CME in patients with Coats'-like RP.

\section{Conflict of interest}

The authors declare no conflict of interest.

\section{References}

1 Zamorani G. Una rara associazone di retinite di coats con retinite pigmentosa. Gior Ital Oftalmol 1956; 9: 429-443.

2 Khan JA, Ide CH, Strickland MP. Coat's-type retinitis pigmentosa. Surv Ophthalmol 1988; 32(5): 317-332.

3 den Hollander AI, Heckenlively JR, van den Born LI, de Kok YJ, van der Velde-Visser SD, Kellner U et al. Leber congenital amaurosis and retinitis pigmentosa with coats-like exudative vasculopathy are associated with mutations in the Crumbs homologue 1 (CRB1) gene. Am J Hum Genet 2001; 69: 198-203.

4 Lee SY, Yoon YH. Pars plana vitrectomy for exuduative retinal detachment in coats-type retinitis pigmentosa. Retina 2004; 24(3): 450-452.

5 Kan E, Yilmaz T, Aydemir O, Güler M, Kurt J. Coats-like retinitis pigmentosa: reports of three cases. Clin Ophthalmol 2007; 1(2): 193-198.

\section{G De Salvo', M Gemenetzi ${ }^{1}$, AJ Luff ${ }^{1}$ and AJ Lotery ${ }^{1,2}$}

${ }^{1}$ Eye Unit, Southampton General Hospital, Southampton, UK

${ }^{2}$ School of Medicine, Southampton University,

Southampton, UK

E-mail: A.J.Lotery@soton.ac.uk

Eye (2011) 25, 821-822; doi:10.1038/eye.2011.25; published online 25 February 2011
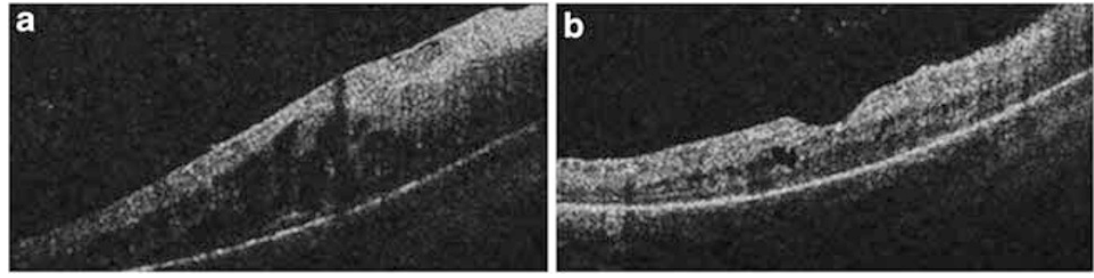

Figure 3 (a) Right eye: 3D-OCT before cryotherapy demonstrates significant cystoid macular oedema and signs of an epiretinal membrane (ERM) (b) Right eye: 3D OCT after cryotherapy demonstrating mild residual cystoid macular oedema and ERM. 